\title{
Discrete supersymmetries of the Schrödinger equation and non-local exactly solvable potentials ${ }^{1}$
}

\author{
Boris F. Samsonov ${ }^{1}$ and A. A. Suzko ${ }^{2}$ \\ ${ }^{1}$ Department of Quantum Field Theory, Tomsk State University \\ 36 Lenin Ave., 634050 Tomsk, Russia, e-mail: samsonov@phys.tsu.ru \\ ${ }^{2}$ Joint Institute for Nuclear Research, 141980 Dubna, Russia, e-mail: suzko@cv.jinr.ru
}

\begin{abstract}
Using an isomorphism between Hilbert spaces $L^{2}$ and $\ell^{2}$ we consider Hamiltonians which have tridiagonal matrix representations (Jacobi matrices) in a discrete basis and an eigenvalue problem is reduced to solving a three term difference equation. Technique of intertwining operators is applied to creating new families of exactly solvable Jacobi matrices. It is shown that any thus obtained Jacobi matrix gives rise to a new exactly solvable non-local potential of the Schrödinger equation. We also show that the algebraic structure underlying our approach corresponds to supersymmetry. Supercharge operators acting in the space $\ell^{2} \times \ell^{2}$ are introduced which together with a matrix form of the superhamiltonian close the simplest superalgebra.
\end{abstract}

\section{Introduction}

Supersymmetric quantum mechanics (SUSY QM) first introduced by Witten [1] as a simplest supersymmetric model of the quantum field theory represents now a fast progressing field of modern theoretical physics (see recent books [2]). As it has been understood after the work by Andrianov et al [3], the SUSY QM may be considered as another formulation of Darboux transformation method well-known in mathematics from the original paper by Darboux [4, book by Ince [5], and book by Matveev and Salle [6] where this method is widely used in the context of the soliton theory. An essential ingredient of the method is a particular choice of a transformation operator (or intertwining operator [7]) in the form of a differential operator (see e.g. [8]) which intertwines two Hamiltonians and relates their eigenfunctions. This approach being applied in quantum theory allows one to generate a huge family of exactly solvable local potentials starting with a given exactly solvable local potential.

\footnotetext{
${ }^{1}$ Published in Phys. Lett. A 302 (2002) 234-241
} 
The technique of transformation operators, first proposed by Delsart [9] (he calls them transmutation operators, see also e.g. [7]) in connection with generalized translation operators, is essentially based on intertwining relations, which have an universal character [10]. It is applicable not only to differential equations (like the Schrödinger equation) but also to difference equations [11, 12, 6] which, in the simplest case, represent eigenvalue problems for Jacobi matrices. Intertwining operators are widely used in the context of soliton solutions of nonlinear lattice equations (see [6] and references therein).

It is known that spectral problems for finite-difference (or discrete) Schrödinger equations have much in common with the theory of orthogonal polynomials [13. The Darboux transformation method gives new insight to this theory and is very fruitful in establishing new properties and deeper understanding some known relations [14. As it was shown in [10], the continuous Darboux transformation is intimately related with quantum inverse scattering problem. A discrete version of this problem has been considered in [15] (for a review see e.g, [16]) but its relationship with discrete Darboux transformations is unknown to the authors. We note that considerable attention has been drown to the non-Hermitian form of the problem [6, 11, 12, 14], while in quantum mechanics one usually uses self-adjoint Hamiltonians. The study of the Hermitian problem has been just started [17. We thus notice that the discrete version of the Darboux transform though ascending to Christoffel [11] and Geronimus [12] works is developed much less than the continuous one.

This paper is aimed to partially fill in this gap. We apply the intertwining relation to a discrete eigenvalue problem for a Jacobi matrix. This allows us to generate a family of new exactly solvable Jacobi matrices. Eigenvalue problems for these matrices naturally appear in quantum mechanics when a discrete basis is used for solving the Schrödinger equation. In particular, there exist exactly solvable Hamiltonians represented by Jacobi matrices [18]. We show that new Jacobi matrices define new exactly solvable potentials of a non-local nature. Moreover, when two lattice eigenvalue problems are written as a single matrix problem, nilpotent difference supercharges may be introduced to map the solutions of these problems to each other. We also show that these supercharges together with a difference superhamiltonian close the simplest superalgebra. We associate these supercharges with discrete supersymmetries of the Schrödinger equation.

\section{Intertwining in $\ell^{2}$-space}

Consider self-adjoint Hamiltonians $h_{0}$ and $h_{1}$ defined in a Hilbert space $H=L^{2}(\mathbb{R})$. We do not exclude the presence of a continuum spectrum and if necessary consider them as defined in a wider space $H_{-}$of linear functionals over $H_{+}, H_{+} \subset H \subset H_{-}$, where $H_{-}$and $H_{+}$are the Hilbert-Schmidt equipment of the space $H$ (so called Gel'fand triplet, see e.g. [19]) without 
especially mentioning it. Let $\left|\psi_{E}\right\rangle$ and $\left|\widetilde{\psi}_{E}\right\rangle$ be eigenkets of $h_{0}$ and $h_{1}$ respectively with an eigenvalue $E, h_{0}\left|\psi_{E}\right\rangle=E\left|\psi_{E}\right\rangle, h_{1}\left|\widetilde{\psi}_{E}\right\rangle=E\left|\widetilde{\psi}_{E}\right\rangle$, and $|n\rangle$ be a basis (orthogonal or not) in $H$. The basis $|n\rangle$ is supposed to be such that the action of both $h_{0}$ and $h_{1}$ takes the form of three term relations:

$$
\begin{aligned}
& h_{0}|n\rangle=a_{n}|n-1\rangle+a_{n+1}|n+1\rangle+q_{n}|n\rangle \\
& h_{1}|n\rangle=\widetilde{a}_{n}|n-1\rangle+\widetilde{a}_{n+1}|n+1\rangle+\widetilde{q}_{n}|n\rangle .
\end{aligned}
$$

We assume that $n$ takes positive integers including zero, $n=0,1,2, \ldots$ and $a_{0}=\widetilde{a}_{0}=0$.

Denote by $\psi_{n}=\psi_{n}(E)$ and $\widetilde{\psi}_{n}=\widetilde{\psi}_{n}(E)$ the Fourier coefficients of $\left|\psi_{E}\right\rangle$ and $\left|\widetilde{\psi}_{E}\right\rangle$ in terms of the basis $|n\rangle$ respectively:

$$
\left|\psi_{E}\right\rangle=\sum_{n} \psi_{n}|n\rangle, \quad\left|\widetilde{\psi}_{E}\right\rangle=\sum_{n} \widetilde{\psi}_{n}|n\rangle
$$

Equations (11) and (2) define the action of $h_{0}$ and $h_{1}$ on any $|\psi\rangle=\sum c_{n}|n\rangle \in H$ where the sum contains a finite number of items. (They form a finite set in $H$.) An eigenket of a Hamiltonian belongs usually to a wider domain which can be specified in terms of its Fourier coefficients over a basis. We will not discuss these mathematical subtleties here and shall simply suppose that all Fourier series converge and when an operator acts on a series it may be moved through the sign of sum. Thus, having (11) and (2) in mind we can act by $h_{0}$ and $h_{1}$ on $\left|\psi_{E}\right\rangle$ and $\left|\widetilde{\psi}_{E}\right\rangle$ given by (3) respectively. As a result we obtain the Fourier series

$$
h_{0}\left|\psi_{E}\right\rangle=\sum_{n}\left(h_{0} \psi\right)_{n}|n\rangle, \quad h_{1}\left|\widetilde{\psi}_{E}\right\rangle=\sum_{n}\left(h_{1} \widetilde{\psi}\right)_{n}|n\rangle
$$

with the Fourier coefficients

$$
\begin{aligned}
\left(h_{0} \psi\right)_{n} & =a_{n} \psi_{n-1}+a_{n+1} \psi_{n+1}+q_{n} \psi_{n}, \\
\left(h_{1} \widetilde{\psi}\right)_{n} & =\widetilde{a}_{n} \widetilde{\psi}_{n-1}+\widetilde{a}_{n+1} \widetilde{\psi}_{n+1}+\widetilde{q}_{n} \widetilde{\psi}_{n} .
\end{aligned}
$$

Note that these quantities depend on the energy $E$ but we do not indicate this to avoid cumbersome notations. The eigenvalue problems for $h_{0}$ and $h_{1}$ are reduced now to the second order three-term finite difference equations

$$
\begin{aligned}
& a_{n} \psi_{n-1}+a_{n+1} \psi_{n+1}+q_{n} \psi_{n}=E \psi_{n} \\
& \widetilde{a}_{n} \widetilde{\psi}_{n-1}+\widetilde{a}_{n+1} \widetilde{\psi}_{n+1}+\widetilde{q}_{n} \widetilde{\psi}_{n}=E \widetilde{\psi}_{n} .
\end{aligned}
$$

Note that the space of sequences $\left\{\psi_{n}\right\}$ with an appropriately defined inner product is known as the space $\ell^{2}$ which also is a Hilbert space (if the space is not complete, it always may be enlarged to become a Hilbert space). If necessary this space may also be equipped with $\ell_{ \pm}^{2}, \ell_{+}^{2} \subset \ell^{2} \subset \ell_{-}^{2}$. The meaning of $\ell_{ \pm}^{2}$ is the same as $H_{ \pm}$. Eqs. (50) and (66) define matrix representations of the Hamiltonians $h_{0}$ and $h_{1}$. 
A second order finite difference equation like a second order differential equation has two linearly independent solutions. This means that for a fixed $E$ one has a two-dimensional linear space of solutions to Eq.(17) (and respectively (8) ). When the spectrum of a Hamiltonian is non-degenerate and the energy is fixed, in this space may exist a unique (up to normalization) element that can be associated with an eigenvector of $h_{0}$. For a bound state eigenvalue $E$ it may be selected from the condition

$$
\left\langle h_{0} \psi_{E} \mid h_{0} \psi_{E}\right\rangle=\sum_{n, k}\left(h_{0} \psi\right)_{k}^{*}\left(h_{0} \psi\right)_{n}\langle k \mid n\rangle<\infty .
$$

We use "*" to distinguish complex conjugate quantities. For a continuous spectrum this value may diverge like the Dirac $\delta$ function,

$$
\left\langle h_{0} \psi_{E} \mid h_{0} \psi_{E^{\prime}}\right\rangle=\sum_{n, k}\left(h_{0} \psi\right)_{k}^{*}(E) \cdot\left(h_{0} \psi\right)_{n}\left(E^{\prime}\right)\langle k \mid n\rangle \sim \delta\left(E-E^{\prime}\right) .
$$

In these equations $\left(h_{0} \psi\right)_{n} \equiv\left(h_{0} \psi\right)_{n}(E)$ are defined in (5). Note also that the continuous spectrum may be two-fold degenerate. In this case any solution of Eq. (77) has a physical meaning. The right-hand sides of Eqs. (51) and (6) define the action of the operators $h_{0}$ and $h_{1}$ in the space $\ell^{2}$, e.g. $h_{0}\left\{\psi_{n}\right\} \equiv\left\{\left(h_{0} \psi\right)_{n}\right\}$.

Denote $\widehat{\psi}_{n}$ another solution of Eq. (17) corresponding to the same energy $E$. Then excluding $q_{n}$ from eigenvalue equations for $\psi_{n}$ and $\widehat{\psi}_{n}$, one arrives at the equation

$$
a_{n}\left(\widehat{\psi}_{n} \psi_{n-1}-\widehat{\psi}_{n-1} \psi_{n}\right)=a_{n+1}\left(\widehat{\psi}_{n+1} \psi_{n}-\widehat{\psi}_{n} \psi_{n+1}\right)
$$

which gives us a discrete analogue of the Wronskian as a combination of $\psi_{n}$ and $\widehat{\psi}_{n}$ independent of the discrete variable $n$

$$
W\left(\widehat{\psi}_{n}, \psi_{n}\right)=a_{n}\left(\widehat{\psi}_{n} \psi_{n-1}-\widehat{\psi}_{n-1} \psi_{n}\right)=w_{0}=\operatorname{const}(n)
$$

When $w_{0}$ and $\psi_{n}$ are fixed, this equation is a recursion relation for $\widehat{\psi}_{n}$ which has the following solution

$$
\widehat{\psi}_{n}=\frac{\widehat{\psi}_{0}}{\psi_{0}} \psi_{n}+\sum_{k=1}^{n} \frac{w_{0} \psi_{n}}{a_{k} \psi_{k} \psi_{k-1}} .
$$

For solutions $\psi_{n}$ and $\xi_{n}$ of (7) with different eigenvalues $E$ and $\lambda$ respectively similar calculation gives a recursion relation for $W\left(\xi_{n}, \psi_{n}\right) \equiv W_{n}$,

$$
W_{n+1}=W_{n}+(\lambda-E) \xi_{n} \psi_{n}
$$

which may be easily iterated to become

$$
W_{n+1}=W_{1}+(\lambda-E) \sum_{k=1}^{n} \xi_{k} \psi_{k} .
$$


Let us suppose that the eigenkets of $h_{0},\left|\psi_{E}\right\rangle$, are known, i.e. one knows solutions of Eq. (71). Then the eigenvalue problem for $h_{1}$, i.e. the search for solutions of (8)), may be replaced by the search for an intertwiner $L$ defined as

$$
L h_{0}=h_{1} L .
$$

Once $L$ is found, the eigenkets $\left|\widetilde{\psi}_{E}\right\rangle$ are obtained by applying $L$ to $\left|\psi_{E}\right\rangle,\left|\widetilde{\psi}_{E}\right\rangle=L\left|\psi_{E}\right\rangle$.

Let us consider a particular anzats for $L$ assuming that it is defined in terms of the basis $|n\rangle$ as follows:

$$
L|n\rangle=A_{n}|n-1\rangle+B_{n}|n\rangle .
$$

Then the Fourier coefficients $\widetilde{\psi}_{n}=L \psi_{n} \equiv(L \psi)_{n}$ of $|\widetilde{\psi}\rangle=L|\psi\rangle=\sum_{n} \widetilde{\psi}_{n}|n\rangle$ are expressed in terms of $\psi_{n}$ :

$$
\widetilde{\psi}_{n}=(L \psi)_{n}=A_{n+1} \psi_{n+1}+B_{n} \psi_{n} .
$$

To determine $A_{n}, B_{n}$ defining $L$ and $\widetilde{a}_{n}, \widetilde{q}_{n}$ defining $h_{1}$, we shall use the intertwining relation (14). The successive application of (15) and (16) gives

$$
L\left(h_{0} \psi\right)_{n}=A_{n+1}\left(a_{n+2} \psi_{n+2}+q_{n+1} \psi_{n+1}+a_{n+1} \psi_{n}\right)+B_{n}\left(a_{n+1} \psi_{n+1}+q_{n} \psi_{n}+a_{n} \psi_{n-1}\right)
$$

whereas the formulae (16) and (6) yields

$$
h_{1}(L \psi)_{n}=\widetilde{a}_{n+1}\left(A_{n+2} \psi_{n+2}+B_{n+1} \psi_{n+1}\right)+\widetilde{q}_{n}\left(A_{n+1} \psi_{n+1}+B_{n} \psi_{n}\right)+\widetilde{a}_{n}\left(A_{n} \psi_{n}+B_{n-1} \psi_{n-1}\right)
$$

from which one finds the following system of equations:

$$
\begin{aligned}
& A_{n} a_{n+1}=A_{n+1} \widetilde{a}_{n}, \\
& B_{n} a_{n}=B_{n-1} \widetilde{a}_{n}, \\
& A_{n+1} q_{n+1}+B_{n} a_{n+1}=B_{n+1} \widetilde{a}_{n+1}+A_{n+1} \widetilde{q}_{n}, \\
& A_{n+1} a_{n+1}+B_{n} q_{n}=\widetilde{a}_{n} A_{n}+B_{n} \widetilde{q}_{n} .
\end{aligned}
$$

Having found $\widetilde{a}_{n}$ from (18) and replaced it in (17), one can "integrate" the latter equation to get

$$
B_{n}=A \frac{a_{n+1}}{A_{n+1}}
$$

where $A$ denotes an "integration constant". After excluding $\widetilde{q}_{n}, \widetilde{a}_{n}$ and $B_{n}$ from (19) and (201) one obtains the equation for $A_{n}$

$$
q_{n}-\frac{A a_{n+1}}{A_{n+1}^{2}} a_{n+1}-\frac{A_{n}^{2}}{A a_{n}} a_{n}=q_{n+1}-\frac{A a_{n+2}}{A_{n+2}^{2}} a_{n+2}-\frac{A_{n+1}^{2}}{A a_{n+1}} a_{n+1} .
$$

Clearly, both left- and right-hand sides of this equation are independent on $n$. Then denoting this quantity by $\lambda$ and introducing a new variable $\xi_{n}$

$$
\frac{\xi_{n-1}}{\xi_{n}}=-\frac{A_{n}^{2}}{A a_{n}}
$$


one arrives at the equation for $\xi_{n}$ :

$$
\xi_{n-1} a_{n}+\xi_{n+1} a_{n+1}+\left(q_{n}-\lambda\right) \xi_{n}=0
$$

The latter equation is nothing but the initial eigenvalue problem (17) which is supposed to be solved. We thus shall suppose that the sequences $\left\{\xi_{n}(\lambda)\right\}$ and $\left\{\widehat{\xi}_{n}(\lambda)\right\}$ being linearly independent solutions of (24) for a fixed value of $\lambda$ are known. When $\lambda=E$ is a point of the spectrum of $h_{0}$, there exists their linear combination which gives the ket $\left|\psi_{E}\right\rangle$ in $n$ representation for non-degenerate $E$. For two-fold degenerate $E$ both $\left\{\xi_{n}(E)\right\}$ and $\left\{\widehat{\xi}_{n}(E)\right\}$ define two linearly independent kets.

Having found $A_{n}$ and $B_{n}$ :

$$
A_{n}=\left[-A a_{n} \xi_{n-1} / \xi_{n}\right]^{1 / 2}, \quad B_{n}=-\left[-A a_{n+1} \xi_{n+1} / \xi_{n}\right]^{1 / 2}
$$

one gets solutions $\widetilde{\psi}_{n}(E)$ of the transformed equation (8)

$$
\begin{gathered}
\widetilde{\psi}_{n}(E)=(L \psi)_{n}(E)=\left[\frac{-A a_{n+1}}{\xi_{n} \xi_{n+1}}\right]^{1 / 2}\left(\xi_{n} \psi_{n+1}(E)-\xi_{n+1} \psi_{n}(E)\right) \\
\left(h_{1} \widetilde{\psi}\right)_{n}(E)=E \widetilde{\psi}_{n}(E) .
\end{gathered}
$$

From (18) one obtains the elements $\widetilde{a}_{n}$

$$
\widetilde{a}_{n}=\frac{1}{\xi_{n}}\left[a_{n} a_{n+1} \xi_{n+1} \xi_{n-1}\right]^{1 / 2}
$$

and from (19) and (20) one has two equivalent expressions for $\widetilde{q}_{n}$ :

$$
\widetilde{q}_{n}=q_{n}+a_{n} \frac{\xi_{n-1}}{\xi_{n}}-a_{n+1} \frac{\xi_{n}}{\xi_{n+1}}=q_{n+1}-a_{n+1} \frac{\xi_{n+1}}{\xi_{n}}+a_{n+2} \frac{\xi_{n+2}}{\xi_{n+1}} .
$$

The formula (26) defines the sequence $\left\{\widetilde{\psi}_{n}(E)\right\}$ for any $E \neq \lambda$. When $E=\lambda$, it gives zero, $(L \xi)_{n}=0$, but we can find a solution of Eq. (8) at $E=\lambda$ by acting with $L$ to another solution of Eq. (24), $\widehat{\xi}_{n}$, which may be found using (11) with the replacement $\psi \rightarrow \xi$. Thus we have the sequence $\left\{\eta_{n}\right\}$ given by

$$
\eta_{n}=(L \widehat{\xi})_{n}=\left[a_{n+1} \xi_{n} \xi_{n+1}\right]^{-1 / 2}, \quad\left(h_{1} \eta\right)_{n}=\lambda \eta_{n}
$$

where inessential constant $w_{0}$ is omitted. Another solution $\widetilde{\eta}_{n}$ of Eq. (8) at $E=\lambda$ may be found by appropriately changed formula (11)

$$
\widehat{\eta}_{n}=\eta_{n}\left[\widehat{\eta}_{0} / \eta_{0}+w_{0} \sum_{k=1}^{n} \xi_{k}^{2}\right], \quad\left(h_{1} \widehat{\eta}\right)_{n}=\lambda \widehat{\eta}_{n}
$$

So, we know all solutions of the transformed discrete equation (8) with $\widetilde{a}_{n}$ and $\widetilde{q}_{n}$ given by (28) and (29) provided all solutions of (17) are known. When $E \neq \lambda$, they are given by 
(26) and for $E=\lambda$ the formulae (30) and (31) should be used. Note that the fact that the sequence $\left\{\widetilde{\psi}_{n}\right\}=\left\{(L \psi)_{n}\right\}$ satisfies the difference equation (8) means that in the space $\ell^{2}$ the intertwining relation $L h_{0}=h_{1} L$ holds where the action of $h_{0}$ on a sequence is defined by (5) and the action of $L$ is defined by (16) with $A_{n}$ and $B_{n}$ from (25).

The most popular bases in quantum mechanics are orthonormal bases, $\left\langle n \mid n^{\prime}\right\rangle=\delta_{n, n^{\prime}}$. For such a basis the action of the operator $L^{+}$, Hermitian conjugate to $L$ with respect to the inner product, on any finite vector $|\widetilde{\psi}\rangle=\sum_{n} \widetilde{\psi}_{n}|n\rangle$ where the sum contains only a finite number of items can be easily found. For this purpose one first notices that for an $L$ given by (15) one has $\left\langle n^{\prime} \mid L n\right\rangle=A_{n} \delta_{n^{\prime}, n-1}+B_{n} \delta_{n^{\prime}, n}=A_{n^{\prime}+1} \delta_{n^{\prime}+1, n}+B_{n} \delta_{n^{\prime}, n}$. Now from the condition $\left\langle n^{\prime} \mid L n\right\rangle=\left\langle L^{+} n^{\prime} \mid n\right\rangle$ one deduces that $L^{+}|n\rangle=A_{n+1}^{*}|n+1\rangle+B_{n}^{*}|n\rangle$ from which it follows that

$$
L^{+}|\widetilde{\psi}\rangle=\sum_{n}\left(L^{+} \widetilde{\psi}\right)_{n}|n\rangle, \quad\left(L^{+} \widetilde{\psi}\right)_{n}=A_{n}^{*} \widetilde{\psi}_{n-1}+B_{n}^{*} \widetilde{\psi}_{n}
$$

with $A_{n}$ and $B_{n}$ given in (25). Since the Hamiltonians $h_{0}$ and $h_{1}$ are self-adjoint, the conjugate operator $L^{+}$satisfies the conjugate intertwining relation $h_{0} L^{+}=L^{+} h_{1}$ which means that $L^{+}$realizes the transformation in the opposite direction, from solutions of (8) to solutions of (7) but it is not inverse to $L$. We shall find the superpositions $L^{+} L$ and $L L^{+}$in the next section.

For a self-adjoint Hamiltonian it is natural to suppose that $a_{n}$ and $q_{n}$ are real for all $n=0,1, \ldots$. This implies that for a real $\lambda$ all $\xi_{n}$ and $\widehat{\xi}_{n}$ may be chosen real which from now on will be assumed. This means that the variables $A_{n}$ and $B_{n}$ defined by (25) may become complex only owing to the constant $-A$. Since the operator $L^{+}$is well-defined for any finite element from $\ell^{2}$, it is not difficult to convince ourselves that $\left(L^{+} \eta\right)_{n}=0$ and $\left(L^{+} \widehat{\eta}\right)_{n}=-w_{0} \sqrt{-A^{*}} \xi_{n}$. Since the usual condition for a Wronskian $w_{0}$ of two solutions belonging to the same energy is $w_{0}=1$, the natural choice for $A$ is $A=-1$. In this case one recovers a discrete analogue of the continuous Darboux transform $\left(L^{+} \widehat{\eta}\right)_{n}=-\xi_{n}$.

So, we see that the discrete Darboux operators $L$ and $L^{+}$realize a one-to-one mapping of the spaces of solutions of discrete Schrödinger equations (77) and (8) for any $E \neq \lambda$. Moreover, this mapping may be continued to $E=\lambda$ by putting $\xi_{n} \longleftrightarrow \widehat{\eta}_{n}$ and $\widehat{\xi}_{n} \longleftrightarrow \eta_{n}$ and considering this as a linear mapping. We conclude hence that the knowledge of all solutions of the initial equation (17) provides the knowledge of all solutions of the transformed equation (8) and, in particular, one can get all "physical" solutions in the usual quantum mechanical sense. For this purpose one notices first that the norm of the vector $\widetilde{\psi}=\left\{\widetilde{\psi}_{n}\right\}=L \psi=\left\{L \psi_{n}\right\}$ is proportional to the norm of the vector $\psi=\left\{\psi_{n}\right\},\langle\widetilde{\psi} \mid \widetilde{\psi}\rangle=\sum_{n} \widetilde{\psi}_{n}^{*} \widetilde{\psi}_{n}=\sum_{n} \psi_{n}^{*}\left(L^{+} L \psi\right)_{n}=$ $(E-\lambda) \sum_{n} \psi_{n}^{*} \psi_{n}=(E-\lambda)\langle\psi \mid \psi\rangle$ where the use of the factorization property has been made. This means that all "physical" solutions of (17) with $E \neq \lambda$ are mapped by $L$ onto "physical" solutions of (8). It is evident that the inverse statement is also true but instead of $L$ one has to use $L^{+}$. To find all "physical" solutions of (8), it remains hence to analyze the sequences 
with eigenvalue $E=\lambda$ forming a 2-dimensional space spanned by $\left\{\eta_{n}\right\}$ and $\left\{\widehat{\eta}_{n}\right\}$ which is not a difficult problem.

As a final remark of this section we note that the formulae (16) and (32) define operators $L$ and $L^{+}$only on finite elements from $H$ (finite linear combinations of basis vectors $|n\rangle$ ). But using the technique of polar decompositions of closed operators in terms of quasi-projectors developed in [20] for a continuous basis, one can extend this action such that the operators $L$ and $L^{+}$become mutually conjugated with respect to the inner product in $H$ with well-defined domains of definitions.

\section{Discrete supersymmetries of the Schrödinger equation}

The usual supersymmetry of the continuous Schrödinger equation is based on factorization properties of Darboux transformation operators (see e.g. [8]). Let us find their counterparts for the discrete equations (7) and (8). For this purpose, let us consider the action of the superposition of $L$ and $L^{+}$on a ket-vector $|\psi\rangle$. Using (16) and (32) one finds

$$
\begin{gathered}
L^{+} L|\psi\rangle=\sum_{n}\left(L^{+} L \psi\right)_{n}|n\rangle \\
\left(L^{+} L \psi\right)_{n}=A_{n}^{*} B_{n-1} \psi_{n-1}+B_{n}^{*} A_{n+1} \psi_{n+1}+\left(\left|A_{n}\right|^{2}+\left|B_{n}\right|^{2}\right) \psi_{n}
\end{gathered}
$$

where $\psi_{n}$ are Fourier coefficients of a vector $|\psi\rangle \in H$ over the basis $|n\rangle$. If for the sake of definiteness we assume that $\lambda<q_{n}$ for all $n$, then from Eq. (24) it follows that both $a_{n} \xi_{n-1} / \xi_{n}<0$ and $a_{n+1} \xi_{n+1} / \xi_{n}<0$ which permits us evaluate absolute values in (33). Finally, using once again the equation for $u_{n}$ (24), one gets

$$
\left(L^{+} L \psi\right)_{n}=|A|\left(\left[h_{0}-\lambda\right] \psi\right)_{n} .
$$

Similar calculations lead to another factorization

$$
\left(L L^{+} \widetilde{\psi}\right)_{n}=|A|\left(\left[h_{1}-\lambda\right] \widetilde{\psi}\right)_{n}
$$

It is necessary to note that these factorizations take place for any sequences $\left\{\psi_{n}\right\}$ and $\left\{\widetilde{\psi}_{n}\right\}$ and the superposition of operators $L$ and $L^{+}$acting in the space of sequences $\ell^{2}$ is defined in the regular way $L^{+} L \psi_{n}=L^{+}(L \psi)_{n}=L^{+} \widetilde{\psi}_{n}=\left(L^{+} \widetilde{\psi}\right)_{n}$. But when $\left\{\psi_{n}\right\}$ is an eigenelement of $h_{0}, h_{0} \psi_{n} \equiv\left(h_{0} \psi\right)_{n}=E \psi_{n}$, one gets from (34) $\left(L^{+} L \psi\right)_{n}=(E-\lambda) \psi_{n}$ where we put $A=-1$. Similarly, when $h_{1} \widetilde{\psi}_{n} \equiv\left(h_{1} \widetilde{\psi}\right)_{n}=E \widetilde{\psi}_{n}$ one obtains using (135) $\left(L L^{+} \widetilde{\psi}\right)_{n}=(E-\lambda) \widetilde{\psi}_{n}$

We can now imitate supercharge operators by introducing nilpotent difference matrix operators

$$
\mathbb{Q}=\left(\begin{array}{ll}
0 & 0 \\
L & 0
\end{array}\right), \quad \mathbb{Q}^{+}=\left(\begin{array}{cc}
0 & L^{+} \\
0 & 0
\end{array}\right)
$$


acting in a vector space of 2-component column-vectors

$$
\left\{\Psi_{n}\right\}=\left(\begin{array}{c}
\left\{\psi_{n}\right\} \\
\left\{\widetilde{\psi}_{n}\right\}
\end{array}\right),
$$

where $\psi_{n}$ and $\widetilde{\psi}_{n}$ are elements of arbitrary sequences from $\ell^{2}$. The superhamiltonian

$$
\mathbb{H}=\left(\begin{array}{cc}
h_{0} & 0 \\
0 & h_{1}
\end{array}\right)
$$

together with supercharges (36) close a simplest superalgebra

$$
[\mathbb{Q}, \mathbb{H}]=\left[\mathbb{Q}^{+}, \mathbb{H}\right]=0, \quad \mathbb{Q}^{2}=\left(\mathbb{Q}^{+}\right)^{2}=0, \quad\left\{\mathbb{Q}, \mathbb{Q}^{+}\right\}=\mathbb{H}-\lambda \mathbb{I}
$$

where $\mathbb{I}$ is the $2 \times 2$ unity matrix. Here commutation relations follow from the intertwining relation for $L, L^{+}, h_{0}$ and $h_{1}$ and the anticommutation relation is an implication of the factorization properties for these operators. The discrete eigenvalue problem for the superhamiltonian $\mathbb{H}$ is nothing but the collection of either equations (77) and (8) in a single matrix equation. Since the operators $\mathbb{Q}$ and $\mathbb{Q}^{+}$from $(\underline{39})$ act in the space $\ell^{2}$, we associate them with discrete supersymmetries of the Schrödinger equation.

\section{New non-local exactly solvable potentials}

Now we shall apply the technique of discrete SUSY (or equivalently discrete Darboux transformations) to generating non-local exactly solvable potentials.

Let us consider the free particle Hamiltonian $h_{0}=p_{x}^{2}=-d^{2} / d x^{2}$. Since the momentum operator may be expressed in terms of the harmonic oscillator creation $a^{+}=i d / d x+i x / 2$ and annihilation $a=i d / d x-i x / 2$ operators $p_{x}=-\left(a+a^{+}\right) / 2$, the Hamiltonian $h_{0}$ is a quadratic form of $a$ and $a^{+}, h_{0}=\left(a+a^{+}\right)^{2} / 4$. Therefore, the action of $h_{0}$ on the oscillator basis $|n\rangle$ which in coordinate representation looks like

$$
\psi_{n}(x)=\langle x \mid n\rangle=(-i)^{n}\left(n ! 2^{n} \sqrt{2 \pi}\right)^{-1 / 2} e^{-x^{2} / 4} H_{n}(x / \sqrt{2})
$$

takes the form of a three term relation

$$
h_{0}|n\rangle=\frac{1}{4} \sqrt{n(n-1)}|n-2\rangle+\frac{1}{4} \sqrt{(n+1)(n+2)}|n+2\rangle+\left(\frac{n}{2}+\frac{1}{4}\right)|n\rangle,
$$

where the use of the well-known properties of the creation and annihilation operators $a|n\rangle=$ $\sqrt{n}|n-1\rangle, a^{+}|n\rangle=\sqrt{n+1}|n+1\rangle$ has been made. Let $\left|\psi_{E}\right\rangle$ be a continuous spectrum eigenket of $h_{0}, h_{0}\left|\psi_{E}\right\rangle=E\left|\psi_{E}\right\rangle$. Then using the self-adjointness of $h_{0}$ we get for the inner product $\psi_{n}=\psi_{n}(E)=\left\langle\psi_{E} \mid n\right\rangle$ the following discrete eigenvalue problem

$$
\begin{aligned}
& a_{n} \psi_{n-2}+a_{n+2} \psi_{n+2}+q_{n} \psi_{n}=E \psi_{n}, \\
& a_{n}=\frac{1}{4} \sqrt{n(n-1)}, \quad q_{n}=\left(\frac{n}{2}+\frac{1}{4}\right) .
\end{aligned}
$$


A "physical" solution to this problem, $\psi_{n}=\psi_{n}(E)$, can be easily obtained since it coincides with the Fourier image of the function (40)

$$
\psi_{n}=2\left(n ! 2^{n} \sqrt{2 \pi}\right)^{-1 / 2} e^{-E} H_{n}(\sqrt{2 E}) .
$$

Here $H_{n}(z)$ are Hermite polynomials. It is not difficult to convince ourselves that Eq. (42) represents another form of the well-known recursion relations for the Hermite polynomials.

The Hamiltonian $h_{0}$ is the kinetic energy operator for $h \equiv h_{1}=h_{0}+V$. Let us consider $V$ such that

$$
V|n\rangle=d_{n}|n-2\rangle+d_{n+2}|n+2\rangle+r_{n}|n\rangle
$$

In coordinate representation this operator is not reduced to the multiplication on a function. We conclude therefore that this is a non-local operator.

Let $\left|\widetilde{\psi}_{E}\right\rangle$ be an eigenvector of $h, h\left|\widetilde{\psi}_{E}\right\rangle=E\left|\widetilde{\psi}_{E}\right\rangle$. Then the eigenvalue problem for the inner products $\widetilde{\psi}_{n}=\widetilde{\psi}_{n}(E)=\left\langle\widetilde{\psi}_{E} \mid \psi_{n}\right\rangle$ is just reduced to the discrete eigenvalue equation (8)) which in our case has the form:

$$
\widetilde{a}_{n} \widetilde{\psi}_{n-2}+\widetilde{a}_{n+2} \widetilde{\psi}_{n+2}+\widetilde{q}_{n} \widetilde{\psi}_{n}=E \widetilde{\psi}_{n}
$$

where

$$
\widetilde{a}_{n}=d_{n}+\frac{1}{4} \sqrt{n(n-1)}, \quad \widetilde{q}_{n}=r_{n}+\frac{n}{2}+\frac{1}{4} .
$$

Consider now a subclass of potentials (45) for which Eq. (46) coincides with the Darboux transform of the Eq. (42). In this case the functions $\widetilde{a}_{n}$ and $\widetilde{q}_{n}$ are defined by Eqs. (28) and (29)

$$
\begin{aligned}
& \widetilde{a}_{n}=\left[a_{n} a_{n+2} \xi_{n-2} \xi_{n+2}\right]^{-1 / 2} / \xi_{n}, \\
& \widetilde{q}_{n}=q_{n+2}-a_{n+2} \xi_{n+2} / \xi_{n}+a_{n+4} \xi_{n+4} / \xi_{n+2}
\end{aligned}
$$

where $a_{n}$ and $q_{n}$ are given in (43) and $\xi_{n}$ is a particular solution of (42) at $E=\lambda<0$, i.e.

$$
\xi_{n}=\left(n ! 2^{n}\right)^{-1 / 2} H_{n}(\sqrt{2 \lambda}) .
$$

Note that these functions are real for $n$ even and purely imaginary for $n$ odd and therefore $\widetilde{a}_{n}$ and $\widetilde{q}_{n}$ are real in either case. Solutions of Eq. (46) are found with the aid of Eq. (26)

$$
\widetilde{\psi}_{n}=\left[\frac{a_{n+2}}{\xi_{n} \xi_{n+2}}\right]^{1 / 2}\left(\xi_{n} \psi_{n+2}-\xi_{n+2} \psi_{n}\right)
$$

which gives us continuous spectrum eigenfunctions for the Hamiltonian $h=h_{0}+V$ with the interaction $V$ given in the form of an infinite tridiagonal matrix

$$
\langle k|V| n\rangle=d_{n} \delta_{k, n-2}+d_{n+2} \delta_{k, n+2}+r_{n} \delta_{k, n}
$$


with $d_{n}$ and $r_{n}$ defined with the aid of (47), (48) and (49). It is not difficult to see that $\eta_{n}$ found by (301) behaves as $n^{-1 / 2}$ and $\widehat{\eta}_{n}$ given by (31) behaves as $n^{3 / 2}$ at large $n$. We conclude hence that no discrete levels in the potential $V$ is created by our method and this is a purely scattering potential.

BFS acknowledges a partial support from the Russian Foundation for Basic Researches. AAS is grateful to Professors H.V. Von Geramb and V. Inozemtzev for useful discussions.

\section{References}

[1] E. Witten, Nucl. Phys. B 185, 513 (1981); 202, 253 (1982).

[2] G. Junker, Supersymmetric Methods in Quantum and Statistical Physics, Springer, Berlin (1996); B.K. Bagchi, Supersymmetry in Quantum and Classical Mechanics, Chapman \& Hall, New York (2001); F. Cooper, A. Khare, U. Sukhatme, Supersymmetry in Quantum Mechanics, World Scientific, Singapore (2001).

[3] A.A. Andrianov, N.V. Borisov, M.V. Ioffe, M.I. Eides, Teor. Mat. Fiz. 61, 17 (1984).

[4] G. Darboux, C.R. Acad. Sci. (Paris) 94, 1456 (1882).

[5] E.L. Ince, Ordinary Differential equations, Dover, New York (1926).

[6] V.B. Matveev, M.A. Salle, Darboux Transformations and Solitons, Springer, Berlin (1991).

[7] B.M. Levitan, Inverse Sturm-Liouville problems, Nauka, Moscow (1984).

[8] V.G. Bagrov and B.F. Samsonov, Theor. Math. Phys. 104, 356 (1995).

[9] J. Delsart, J. Math. Pures et Appl. 17, 213 (1938); C. R. Acad. Sci. (Paris) 206, 178 (1938).

[10] V.E. Zakharov and A.B. Shabat, Funct. Anal. Appl. 8, 226 (1974); Funct. Anal. Appl. 13, 166 (1979).

[11] T.S. Chihara, Introduction to Orthogonal Polynomials, Gordon and Breach, New York (1978); G. Szegö, Orthogonal Polynomials (4th edn.), Providence R, American Mathematical Society (1975).

[12] Ya.I. Geronimus, Izv. Acad. Nauk SSSR 4, 215 (1940).

[13] Yu.M. Berezanskii, Expansions in terms of Eigenfunctions of Selfadjoint Operators, Naukova Dumka, Kiev (1965). 
[14] V. Spiridonov, A. Zhedanov, Meth. Appl. Anal. 2 (4), 369 (1995).

[15] K.M. Case and M. Kac, J. Math. Phys. 14, 594 (1973); K.M. Case, J. Math. Phys. 15, 2166 (1974).

[16] B.N. Zakhariev and A.A. Suzko, Direct and Inverse Problems. (Potentials in Quantum Scattering), Springer-Verlag. Berlin Heidelberg/New York, 2nd ed. (1990)

[17] V. Spiridonov, A. Zhedanov, Annals of Physic, 237, 126 (1995); A.A. Suzko, Physics of Atomic Nuclei, 65, 1553 (2002).

[18] H.A. Yamani, L. Fishman, J. Math. Phys. 16, 410 (1975).

[19] I.M. Gel'fand and G.E. Shilov, The generalised functions, 1 - 3, Phys.-Math. Publishing State House, Moscow (1957, 1958); I.M. Gel'fand and N. Ya. Vilenkin, The generalised functions, 4., Phys.-Math. Publishing State House, Moscow (1961); F.A. Berezin and M.A. Shubin, The Schrödinger Equation, Kluwer, Dordrecht (1991).

[20] B.F. Samsonov, J. Phys. A: Math. Gen. 33, 591 (2000). 